\title{
SOME CYCLIC FIXED POINT RESULTS FOR CONTRACTIVE MAPPINGS
}

\author{
Stojan Radenovic $^{1}$, Sumit Chandok $^{2 *}$, Wasfi Shatanawi ${ }^{3}$ \\ ${ }^{1}$ Faculty of Mechanical Engineering, University of Belgrade, Serbia. \\ ${ }^{2}$ School of Mathematics, Thapar University, Patiala, India. \\ ${ }^{3}$ Department of Mathematics, Hashemite University, Zarqa, Jordan.
}

\begin{abstract}
In this paper, we obtained some new cyclic type fixed point theorems using $\boldsymbol{C}$-class functions in the framework of metric spaces. Our results extend,

generalize, improve and enrich some recent results in the existing literature.
\end{abstract}

Key words: contractive mappings, cyclic mapping, Cauchy sequence, fixed point, $C$-class function.

MSC: 47H10, 54H25.

\section{INTRODUCTION AND PRELIMINARIES}

Very recently, ((Chandok et al., 2016), Theorem 14.) proved the following result:

Theorem 1.1. ((Chandok et al., 2016), Theorem 1). Let $\mathrm{A}$ and $\mathrm{B}$ be two closed subsets of complete metric spaces $(\mathrm{X}, \mathrm{d})$ such that $A \cap B \neq \varnothing$ and let $T: A \cup B \rightarrow A \cup B$ be a mapping such that $T A \subset B$ and $T B \subset A$. Assume that:

$$
\psi(d(T x, T y)) \leq f(\psi(d(x, y)), \phi(d(x, y))),(1.1)
$$

for all $x \in A$ and $y \in B$, where $f \in C, \psi \in \Psi$, and $\phi \in \phi$. Then $T$ has a unique fixed point in $A \cap B$.

In this paper, using $C$-class functions, we prove some new cyclic type fixed point theorems. Our results extend, generalize, improve and enrich recently ones in existing literature.

First, we recall some notions and properties that will be needed throughout the paper.

Definition 1.1. ((Chandok et al., 2016), Definition $5)$. One can say that $f:[0,+\infty)^{2} \rightarrow \mathbb{R}$ is called $C$ -class function if it is continuous and satisfies the following axioms:

(1) $f(s, t) \leq s$ for all $s, t \geq 0$.

(2) $f(s, t)=s$ implies that either $s=0$ or $t=0$ for all $s, t \geq 0$.

We denote $C$-class functions as $C$.

Example 1.1. ((Chandok et al., 2016), Example 6). The following functions $f:[0,+\infty)^{2} \rightarrow \mathbb{R}$ are elements of $C$.

(1) $f(s, t)=s-t$;

(2) $f(s, t)=k s, k \in(0,1)$;
(3) $f(s, t)=\frac{s}{1+t}$;

(4) $f(s, t)=\frac{\log \left(t+a^{s}\right)}{1+t}, a>1$;

(5) $(s, t)=\frac{\ln \left(1+a^{s}\right)}{2}, a>e$;

(6) $f(s, t)=(s+l)^{\frac{1}{1+t}}-l, l>1$.

Further, let $\psi$ denote the set of all monotone increasing continuous functions $\psi:[0,+\infty) \rightarrow$ $[0,+\infty)$, with $\psi^{-1}(\{0\})=\{0\}$, as well as let $\phi$ denote the set of all continuous functions $\phi$ : $[0,+\infty) \rightarrow[0,+\infty)$, with $\lim _{n \rightarrow \infty} \phi\left(t_{n}\right)=0$ implies $\lim _{n \rightarrow \infty} t_{n}=0$.

In the sequel, let $X$ be a nonempty set, $A_{1}, A_{2}, \ldots, A p$ be its nonempty subsets. Recall that $Y=\cup_{i=1}^{p} A_{i}$ is said to be a cyclic representation of $Y$ with respect to a mapping $T: Y \rightarrow Y$ if:

$$
T\left(A_{1}\right) \subset A_{2}, \ldots, T\left(A_{p}\right) \subset A_{1} .
$$

We will use the following auxiliary result.

Lemma 1.2. ((Radenović, 2015b), Lemma 2.1). Let $(X, d)$ be a metric space, $T: X \rightarrow X$ be a mapping and let $X=\cup_{i=1}^{p} A_{i}$ be a cyclic representation of $X$ w.r.t. T. Assume that:

$$
\lim _{n \rightarrow \infty} d\left(x_{n}, x_{n+1}\right)=0,
$$

where $x_{n+1}=T x_{n}, x_{1} \in A_{1}, n \in \mathbb{N}$. If $\left\{x_{n}\right\}$ is not a Cauchy sequence, then there exist an $\varepsilon>0$ and two sequences $\{n(k)\}$ and $\{m(k)\}$ of positive integers such that $n(k)>m(k)>k$ and the following sequences tend to $\varepsilon^{+}$as $k \rightarrow \infty$ :

Mathematics 


$$
\begin{gathered}
d\left(x_{m(k)-j(k)}, x_{n(k)}\right), d\left(x_{m(k)-j(k)+1}, x_{n(k)}\right), \\
d\left(x_{m(k)-j(k)}, x_{n(k)+1}\right), d\left(x_{m(k)-j(k)+1}, x_{n(k)+1}\right),
\end{gathered}
$$

where $j(k) \in\{1,2, \ldots, p\}$ is chosen so that $n(k)-$ $(m(k)-j(k)) \equiv 1(\bmod p)$, for each $k \in \mathbb{N}$.

If $p=1$ we obtain very significant, important as well as useful result in the framework of metric space for standard fixed point results ((Radenović et al., 2012), Lemma 2.1.). Hence, Lemma 1.2. is its generalization.

\section{MAIN RESULTS}

Our main result is the following improvement of Theorem 1.1. that is, Theorem 14 from (Chandok et al., 2016). It also generalizes several enough known results in existing literature.

Theorem 2.1. Let $(X, d)$ be a complete metric space, and let $Y=\cup_{i=1}^{p} A_{i}$ be a cyclic representation of $Y \subseteq X$ with the respect to a mapping $T: Y \rightarrow Y$, where the sets $A_{i}$ are closed. Assume that:

$$
\psi(d(T x, T y)) \leq f(\psi(d(x, y)), \phi(d(x, y))),
$$

for all $x \in A_{i}, y \in A_{i+1}$, where $f \in C, \psi \in \Psi$, and $\phi \in \phi$. Then, $T$ has a unique fixed point $z \in Y$ and $z \in \cap_{i=1}^{p} A_{i}$. Moreover, each Picard sequence $x_{n}=T^{n} x, x \in Y$ converges to $z$.

Proof. First of all, if $T$ has a fixed point say $z \in Y$, using (1.2), we have $z \in \cap_{i=1}^{p} A_{i}$. (for more details see (Van Dung \& Radenović, 2016), (Kadelburg et al., 2016), (Radenović et al., 2016), (Radenović, 2015a), (Radenović, 2016), (Radenović, 2015b). Further, if $T$ has another fixed point $z_{1} \neq z$, we get by (2.1)

$$
\begin{gathered}
\psi\left(d\left(z, z_{1}\right)\right)=\psi\left(d\left(T z, T z_{1}\right)\right) \leq \\
f\left(\psi\left(d\left(z, z_{1}\right)\right), \phi\left(d\left(z, z_{1}\right)\right)\right) \leq \psi\left(d\left(z, z_{1}\right)\right) .
\end{gathered}
$$

Now from (2.2) follows $f\left(\psi\left(d\left(z, z_{1}\right)\right), \varphi\left(d\left(z, z_{1}\right)\right)\right)$ $=\psi\left(d\left(z, z_{1}\right)\right)$, which implies either $\psi\left(d\left(z, z_{1}\right)\right)=0$ or $\phi\left(d\left(z, z_{1}\right)\right)=0$. Since $z_{1} \neq z$ then both cases follows a contra diction. Hence, if $T$ has a fixed point then it is a unique as well as belongs $\cap_{i=1}^{p} A_{i}$.

Now, to prove that $T$ has a fixed point, take arbitrary $x_{0} \in Y$. It belongs to some of the subsets $A_{i}, i \in\{1, \ldots, p\}$, for example $x_{0} \in A_{1}$. It is clear that then the Picard sequence $x_{n}=T x_{n-1}, n \in \mathbb{N}$, is divided into the following $p$ subsequences, each belonging to some $A_{i}, i \in\{1, \ldots, p\}$ :

$$
\left\{x_{n p}\right\} \subset A_{1},\left\{x_{n p+1}\right\} \subset A_{2}, \ldots,\left\{x_{n p+p-1}\right\} \subset A_{p}
$$

If $x_{k+1}=x_{k}$ for some $k \in \mathbb{N}$ then $x_{k}$ is a unique fixed point of $T$ and it belongs to $\cap_{i=1}^{p} A_{i}$. Therefore, suppose that $x_{n+1} \neq x n$ for each $n$. Further, it can be proved in a standard way (e.g., as in the proof of (Chandok et al., 2016), Theorem 8) that $d\left(x_{n}, x_{n+1}\right)$ is a decreasing sequence and converges to 0 as $n \rightarrow \infty$.

Further, we have to prove that $\left\{x_{n}\right\}$ is a Cauchy sequence. If it is not case, then, using Lemma 1.2, we have that there exist an $\varepsilon>0$ and two sequences $\{n(k)\}$ and $\{m(k)\}$ of positive integers, with $n(k)>$ $m(k)>k$, such that the sequences (1.3) tend to $\varepsilon^{+}$ when $k \rightarrow \infty$. Putting in $(2.1),=x_{m(k)-j(k)}, y=$ $x_{n(k)}$, we get:

$$
\begin{gathered}
\psi\left(d\left(x_{m(k)-j(k)+1}, x_{n(k)+1}\right)\right) f\left(\psi\left(d\left(x_{m(k)-j(k)}, x_{n(k)}\right)\right),\right. \\
\left.\phi\left(d\left(x_{m(k)-j(k)}, x_{n(k)}\right)\right)\right) .
\end{gathered}
$$

Passing to the limit in (2.4) as $k \rightarrow \infty$, we get:

$$
\psi(\varepsilon) \leq f(\psi(\varepsilon), \varphi(\varepsilon)) \leq \psi(\varepsilon),
$$

which implies that $f(\psi(\varepsilon), \phi(\varepsilon))=\psi(\varepsilon)$. According to the property of $C$-class function $f$, we get either $\psi(\varepsilon)=0$ or $\phi(\varepsilon)=0$. In both the cases we obtain that $\varepsilon=0$, which is a contradiction. Therefore, we obtain that Picard sequence $\left\{x_{n}\right\}$ is a Cauchy. Since $(X, d)$ is a complete then $(Y, d)$ is also complete, that is, there exists $z \in Y=\cup_{i=1}^{p} A_{i}$ such that $x_{n} \rightarrow z$ as $n \rightarrow \infty$. This means that $z \in A_{l}$ for some $l \in\{1, \ldots, p\}$ and, hence, $T z \in A_{l+1}$. Consider the subsequence $\left\{x_{n p+l}\right\} \subset A_{l+1}$ of $\left\{x_{n}\right\}$. Applying (2.1), we get:

$$
\begin{gathered}
\psi\left(d\left(x_{n p+l+1}, T z\right)\right)=\psi\left(d\left(T x_{n p+l}, T z\right)\right) \leq \\
f\left(\psi\left(d\left(x_{n p+l}, z\right)\right), \phi\left(d\left(x_{n p+l}, z\right)\right)\right) \leq \\
\psi\left(d\left(x_{n p+l}, z\right)\right),
\end{gathered}
$$

that is, $\psi\left(d\left(x_{n p+l+1}, T z\right)\right) \leq \psi\left(d\left(x_{n p+l}, z\right)\right)$. Since subsequences $\left\{x_{\mathrm{np}+l}\right\}$ and $\left\{x_{n p+l+1}\right\}$ converges to $Z$ also, then passing to the limit as $n \rightarrow \infty$ we get that $T z=z$. The proof of Theorem 2.1. is complete.

Corollary 2.1. Let $(X, d)$ be a complete metric space, and let $T: X \rightarrow X$ be a mapping such that:

$$
\psi(d(T x, T y)) \leq f(\psi(d(x, y)), \phi(d(x, y))),
$$

for all $x, y \in X$, where $f \in C, \psi \in \Psi$, and $\phi \in \phi$. Then, $T$ has a unique fixed point $z \in X$. Moreover, each Picard sequence $x_{n}=T^{n} x, x \in X$ converges to $z$.

Proof. Putting in Theorem 2.1. $A_{i}=X$ for all $i=1, \ldots, p$ we obtain the result. $\square$

Similarly as in (Kadelburg et al., 2016.), (Radenović et al., 2016), (Radenović 2015a), 
(Radenović 2016), (Radenović 2015b), one can easily prove that Theorem 2.1 and Corollary 2.1 are equivalent, that is, Theorem 2.1 holds if and only if Corollary 2.1 holds.

We now announce the following very significant as well as new result with new concept of $C$-class functions:

Theorem 2.2. Theorem 2.1 and Corollary 2.1 are equivalent

Proof. Method as well as ideas for the proof are the same as ones in (Radenović et al., 2016), (Radenović, 2015a), (Radenović, 2016), (Radenović, 2015b).

Remarks 2.1. (a) Putting $p=2, A_{i}=A, A_{2}=B$ (but without assumption $A \cap B \neq \emptyset$ ) we get Theorem 14 from (Chandok et. al., 2016).

(b) Putting $p=2, A_{1}=A, A_{2}=B, f(s, t) s-$ $t, \psi(t)=t, \phi(t)=(1-k) t, k \in(0,1) \quad$ we get Theorem 1.1 from (Kirk et. al., 2003).

For more new details regarding recent results for cyclic cases see ((Van Dung \& Hang, 2016), (Van Dung \& Radenović, 2016), (Kadelburg et al., 2016.), (Radenović et al., 2016), (Radenović, 2015a), (Radenović, 2016), (Radenović, 2015b)).

Finally, we have the following open question: Does the following Theorem holds?

Theorem 2.3. Let $(X, d)$ be a complete metric space, and let $Y=\cup_{i=1}^{p} A_{i}$ be a cyclic representation of $Y \subseteq X$ with the respect to a mapping $T: Y \rightarrow Y$, where the sets $A_{i}$ are closed. Assume that for all $x \in A_{i}$, $y \in A_{i+1}$ there exists $u(x, y) \in\{d(x, y), d(x, f x)$, $\left.d(y, f y), \frac{d(x, f y)+d(y, f x)}{2}\right\}$ such that:

$$
\psi(d(T x, T y)) \leq f(\psi(u(x, y)), \phi(u(x, y))),
$$

where $f \in C, \psi \in \Psi$, and $\phi \in \phi$.Then, $T$ has a unique fixed point $z \in Y$ and $z \in \cap_{i=1}^{p} A_{i}$. Moreover, each Picard sequence $x_{\mathrm{n}}=T^{n} x, x \in Y$ converges to $z$.

\section{ACKNOWLEDGMENT}

The authors are thankful to the anonymous learned referees for very careful reading and valuable suggestions.

\footnotetext{
*E-mail: chandhok.sumit@gmail.com
}

\section{CONFLICT OF INTERESTS}

Authors declare that they have no any conflict of interest regarding the publication of this paper.

\section{REFERENCES}

Chandok, S., Tas, K., \& Hojat Ansari, A., 2016. Some fixed point results for TAC-type contractive mappings, Journal of Function Spaces, 2016, Article ID 1907676, pp. 1-6.

Kadelburg, Z., Radenović, S., \& Vujaković, J., 2016. A note on the paper "Fixed point theorems for cyclic weak contractions in compact metric spaces", Fixed Point Theory Appl. 2016:46, DOI 10.1186/s13663016-0537-0.

Kirk, W., Srinavasan P., \& Veeramani, P., 2003. Fixed points for mapping satisfying cyclical contractive conditions, Fixed Point Theory, 4(1), pp.79-89.

Radenović,S., Došenović, T., Aleksić Lampert, T., \& Golubović, Z., 2016. A note on some recent fixed point results for cyclic contractions in b-metric spaces and an application to integral equations, Applied Mathematics and Computation. 273, pp. 155-164. Doi:10.1016/j.amc.2015.09.089.

Radenović, S., Kadelburg, Z., Jandrlić, D., \& Jandrlić, A., 2012. Some results on weakly contractive maps, Bull. Iran. Math. Soc. 38(3), pp. 625-645.

Radenović, S., 2015a. A note on fixed point theory for cyclic weaker Meir-Keeler function in complete metric spaces, Int. J. Anal. Appl. 7(1), pp. 16-21.

Radenović, S., 2015b. A note on fixed point theory for cyclic $\phi$-contractions, Fixed Point Theory Appl., 2015:189.DOI: 10.1186/s13663-015-0437-8.

Radenović, S., 2016. Some remarks on mappings satisfying cyclical contractive conditions, Afrrika Matematika. 27(1), pp. 291-295. DOI:10.1007/s13370-015-0339-2.

Van Dung, N., \& Hang, V., 2016. Remarks on cyclic contractions in b-metric spaces and applications to integral equations, Revista de la Academia de Ciencias Exactas, Fisicas y Naturales, Serie A. Matematicas, DOI 10.1007/s13398-016-0291-5.

Van Dung, N., \& Radenović, S., 2016, Remarks on theorems for cyclic quasi-contractions, Kragujevac Journal of Mathematics. 40(2), pp. 272-279. 